

\section{DIGITALIZACION EN LA MEDICINA: IMPLICACIONES PARA EL DISEÑO, INNOVACION Y LA INGENIERIA}

\author{
J. Munguia ${ }^{1 *}$ \\ 1: Profesor asistente, Universidad de Newcastle upon Tyne. Escuela de Ingeniería. \\ Newcastle upon Tyne, Reino Unido \\ *Javier.munguia@ncl.ac.uk
}

\begin{tabular}{l}
\hline PALABRAS CLAVE \\
\hline Digitalización \\
Diseño \\
Ingeniería y manufactura \\
digital.
\end{tabular}

\section{RESUMEN}

La aplicación de las tecnologías de diseño computacional, manufactura digital, así como el uso de técnicas de visualización aumentada se encuentra en aumento en los sistemas hospitalarios. Mientras que los hospitales universitarios cuentan con la ventaja de fácil acceso a instalaciones de disciplinas como ingeniería, materiales, diseño o mecatrónica, los hospitales tanto públicos, como privados se enfrentan al reto de tener acceso a nuevas tecnologías que se abren camino en el entorno médico: diseño computarizado, acceso a técnicas de fabricación, software y digitalización de información de radiodiagnóstico. Este artículo recopila experiencias recientes de varias unidades médicas del sistema hospitalario de Newcastle upon Tyne, perteneciente al sistema nacional de salud del Reino Unido (NHS). Los recientes resultados muestran una creciente tendencia hacia la digitalización que tiende a modificar los flujos de trabajo y en general la forma en la que se desarrollan distintos dispositivos médicos dentro del sistema de salud en cuestión.

\section{DIGITAL MEDICINE: IMPLICATIONS ON DESIGN, INNOVATION AND ENGINEERING}

\begin{tabular}{|c|c|}
\hline KEYWORDS & ABSTRACT \\
\hline $\begin{array}{l}\text { Digitalization, } \\
\text { Design } \\
\text { Engineering and digital } \\
\text { manufacturing }\end{array}$ & $\begin{array}{l}\text { The application of digital manufacturing and virtual visualization techniques for } \\
\text { healthcare purposes is on a steady growth, with different medical units such as } \\
\text { anesthetics, genetic medicine, dentistry and medical physics gradually adopting } \\
\text { them on a normal basis. Whilst University-Hospitals have the advantage of } \\
\text { having support from mechanical, bioengineering and design departments, some } \\
\text { common questions arising from other independent healthcare systems wanting to } \\
\text { apply digital technologies are: who can perform the design? What software is } \\
\text { available and what is need it to operate it? This work describes the experience of } \\
\text { different units across the Newcastle Hospitals NHS Foundation Trust on how } \\
\text { digital design and manufacturing technologies are permeating the conventional } \\
\text { Healthcare structure, including an insight in-to how the in-clinic fabrication } \\
\text { possibilities afforded by digital technologies can change the medical devices } \\
\text { supply chain for a variety of purposes. }\end{array}$ \\
\hline
\end{tabular}




\section{INTRODUCCIÓN}

\subsection{Digitalización en Hospitales}

La 'digitalización' en un entorno sanitario es comúnmente asociada con el despliegue de Tecnologías de Información y Comunicaciones (TICs) ya sea por conectividad, automatización o agilización de procedimientos internos e intercambio de sistemas de datos de información de pacientes [1-4]. Un "Hospital Digital" se define como:

"Un servicio médico orientado al usuario que incluye servicios digitales como: diagnóstico, asesoría y tratamiento sin limitaciones de tiempo y espacio" [5]. Sin embargo, existe una digitalización paralela relacionada con el hardware que incluye: desarrollo de dispositivos médicos y electrónicos, modelos de diagnóstico, software de tratamiento de imágenes médicas, impresión 3D o diseño de ingeniería aplicado a implantología.

Desde 2017 el sistema nacional de salud del Reino Unido (NHS) instauró una nueva figura de puesto de trabajo denominada: "Técnico Biomédico 3D", lanzada en el Hospital Swansea Morriston [6]. Esta figura permite a un centro médico reclutar a profesionales de la ingeniería, diseño o manufactura para dedicarse a desarrollos internos en materia de implantes, guías quirúrgicas, así como en la innovación hospitalaria con tecnologías propietarias.

El desarrollo de dichas tecnologías y su creciente penetración en ámbitos sanitarios está motivando un cambio 'digital' en varias disciplinas médicas analizadas a continuación.

\subsection{Ecosistema del 'Hospital Digital'}

La tesis inicial es que un hospital universitario presenta el ecosistema ideal para la digitalización en varios sectores, no solo por la confluencia de disciplinas en un mismo entorno sino por el acceso a equipamiento, personal técnico y académico que favorece la adopción de tecnologías emergentes [7]. La Figura 1 muestra distintas áreas pioneras en adoptar diferentes tipos de 'digitalización' en el sistema hospitalario de Newcastle upon Tyne (NHSTrust).



Figura 1. Unidades médicas asociadas al sistema Newcastle NHS trust y Newcastle University.

\section{EL PROCESO DE DIGITALIZACION}

La digitalización actual incide no solo en la automatización de datos, información de pacientes y bases de datos electrónicas si no que se extiende a la captura, recolección, mantenimiento y procesamiento de información médica en forma de imágenes, conjuntos de datos, e incluso archivos/ficheros con información tridimensional. Esto requiere un perfil profesional distinto, entrenado y con destreza en el uso de dichas herramientas, lo que hemos denominado 'Digital literacy' [8] es decir, actualizar las ocupaciones 
tradicionales considerando el entorno digital del siglo XXI [9].

Por ejemplo, un perfil clásico de 'Técnico en radiología' es el profesional experto en obtención de imágenes diagnósticas y su procesamiento; sin embargo, este perfil será gradualmente ampliado hasta abarcar: el uso de software de segmentación médica tridimensional, extracción de modelos $3 \mathrm{D}$ a partir de imágenes 2D, preparación de modelos virtuales para impresión 3D, edición en software de mallado y superficies 3D. La Tabla 1 especifica las tendencias en digitalización que han tenido lugar en varias disciplinas relacionadas con el entorno Newcastle-NHS.

Tabla 1. Principales disciplinas y transformaciones clave motivadas por la tendencia digital.

\begin{tabular}{|c|c|}
\hline $\begin{array}{l}\text { Bio- } \\
\text { mecánica }\end{array}$ & $\begin{array}{l}\text { - Toma de impresiones de extremidades } \\
\text { superiores/inferiores a través de escáner 3D, } \\
\text { reemplazando moldes de yeso/escayola. } \\
\text { - Análisis de marcha basado en } \\
\text { reconocimiento de imágenes, reemplazando } \\
\text { marcadores táctiles y sensores. } \\
\text { - Diseño de plantillas plantares en software 3D } \\
\text { para mecanizado, reemplazando } \\
\text { termoformado manual. }\end{array}$ \\
\hline $\begin{array}{l}\text { Prótesis/ } \\
\text { órtesis }\end{array}$ & $\begin{array}{l}\text { - Alineamiento y rectificación a partir de } \\
\text { escaneo 3D para muñones de rodilla, brazo y } \\
\text { corsés. } \\
\text { - Análisis de presión plantar en tiempo real. } \\
\text { - Impresión 3D de sockets/muñones } \\
\text { personalizados para prótesis. }\end{array}$ \\
\hline $\begin{array}{l}\text { Radio } \\
\text { diagnóstico }\end{array}$ & $\begin{array}{l}\text { - Segmentación y reconstrucción de imágenes a } \\
\text { partir de datos radiológicos. } \\
\text { - Diseño 3D orgánico (BioCAD). } \\
\text { - Manipulación de información y archivos para } \\
\text { manufactura digital. }\end{array}$ \\
\hline $\begin{array}{l}\text { Odonto- } \\
\text { logía }\end{array}$ & $\begin{array}{l}\text { - Escaneo 3D intraoral reemplazando modelos } \\
\text { de silicona /yeso para molduras. } \\
\text { - Reemplazo de modelos de yeso/ escayola } \\
\text { mediante modelos impresos en resina 3D. } \\
\text { - Alineación dental a través de software, } \\
\text { reemplazando ortodoncia tradicional. } \\
\text { - Diseño de coronas, implantes y análisis de } \\
\text { mordida a partir de modelos 3D en } \\
\text { estereolitografía. }\end{array}$ \\
\hline
\end{tabular}

Los procesos de digitalización descritos tienen una mayor profundidad en diferentes áreas. Por ejemplo, en el campo odontológico, varias facultades universitarias han reemplazado los laboratorios clásicos de modelado y fabricación por laboratorios digitales donde el diseño 3D computarizado reemplaza el pulido y acabado manual de prótesis dentales mientras que la impresión 3D reemplaza procesos clásicos de moldeo, colada de cera o formado en metal [10].

\section{MAPEO DE DIGITALIZACIONES}

Aunque existe evidencia de aplicaciones médicas de la digitalización, diseño y manufactura digital en general desde la década de los 90s [11-12], no ha sido sino hasta los últimos 10 años que el sector médico ha iniciado la adopción gradual de dichas tecnologías en reemplazo de procedimientos convencionales. Diversos estudios documentan el uso de la manufactura digital para la creación de implantes óseos, dispositivos auditivos, arneses de soporte tipo exoesqueletos, implantes mandibulares, e incluso bioimpresión 3D para el cultivo celular, desarrollo de estructuras de andamio (scaffolds) para el análisis de supervivencia de células [12].

Mientras que cada región tendrá un mapeo distinto de tecnologías digitales e interacciones con distintos departamentos, en el sistema de hospitales de Newcastle upon Tyne hemos clasificado la 'digitalización' en tres tipos (Figura 2): 1) Desarrollo de dispositivos médicos, 2) Desarrollo de modelos quirúrgicos y bioimpresión, 3) Desarrollo de modelos para enseñanza y educación. 




Figura 2. Tres líneas principales de digitalización del entorno Universidad/Hospital [13].

Cabe señalar que cada ecosistema Universidad/Hospital tendrá un mapeo de acuerdo a sus áreas de especialidad y capacidad instalada, por ejemplo, mientras que en el 'ecosistema Londres', University College London (UCL) desarrolla procedimientos no-invasivos de bioimpresión junto con su Hospital asociado (Great Ormond Street Institute) [14], en el 'ecosistema Liverpool' se desarrolla tecnología de implantes metálicos impresos en 3D para licenciar al sector privado con actores como Stryker Orthopaedics y Renishaw PLC [15].

\section{CASOS DE ESTUDIO}

\subsection{Desarrollo de dispositivos médicos: máscara CPAP}

Una máscara CPAP (Continuous Positive Airway Pressure) es un dispositivo médico mediante el cual se ofrece un tratamiento de presión positiva en las vías respiratorias para aliviar síntomas relacionados con apnea del sueño, que en su versión más grave puede generar trastornos del sueño, así como problemas respiratorios (Figura 3-Izquierda). Uno de los motivos por los que el tratamiento CPAP resulta inefectivo es por el abandono del mismo por parte del paciente, en respuesta a la falta de confort y dificultades de ajuste.



Figura 3. Paciente utilizando máscara CPAP (Izquierda), escaneo $3 D$ del rostro (Centro), y máscara CPAP digitalizada para análisis de presiones (Derecha) [13].

La escuela de Ingeniería de la Universidad de Newcastle, en conjunto con la Unidad de Función Respiratoria de Freeman Hospital (Newcastle) realizaron un estudio de pacientes para evaluar la posibilidad de personalizar las máscaras CPAP, de manera que a partir de un estudio topológico de características faciales se genere una máscara que se ajuste al contorno exacto del paciente, minimizando el riesgo de abandono del tratamiento (Figura 3 centro/derecha).

Los métodos para el desarrollo de dichas máscaras incluyeron el uso de escaneo 3dimensional (Creaform Go-Scan), software de edición de nube de puntos en 3D (VX Elements) así como el uso de software para realizar mediciones topológicas y diseño 3D (Materialise 3-Matic). La geometría final fue impresa en 3D a manera de prototipo mediante el software CURA para Ultimaker $3+$.

El resultado fue validado por un muestreo de usuarios en donde cada paciente se presentó a una sesión de escaneo 3D, seguido del diseño e impresión 3D de una máscara CPAP personalizada (Figura 4).

La evaluación de confort y ajuste se realizó en comparación con una máscara CPAP convencional (Fisher \& Paykell) así como una máscara genérica impresa en $3 \mathrm{D}$ como elemento de control, con resultados mostrando una clara preferencia de los usuarios hacia el diseño personalizado. 




Figura 4. a) Mascara CPAP estándar, b) Máscara de control y c) Máscara personalizada impresa en $3 D$ [16].

\subsection{Desarrollo de modelos quirúrgicos}

La escuela de Ingeniería (Universidad de Newcastle-UNEW) fue contactada por la Unidad de Neurología del Royal Victoria Infirmary (NHS Hospitals) con el fin de desarrollar un modelo computarizado para la práctica de endoscopía ventricular a partir de imágenes médicas de Tomografía Computarizada, con especial énfasis en la sección craneal.

Las imágenes médicas fueron importadas al software Mimics Innovation Suite (Materialise, Bélgica) y posteriormente segmentadas e interpoladas a un modelo craneal 3D (Figura 5).

El resultado (Figura 6) fue un modelo 3D anatómico y modular que ofrece múltiples puertos para la práctica de la cirugía a partir de diferentes ángulos. La continuación de este proyecto incluirá la impresión 3D de los modelos, así como la segmentación de otras áreas cerebrales a partir de imágenes de resonancia magnética.



Figura 5. Vista general del área de segmentación en el software Mimics de Materialise. a)

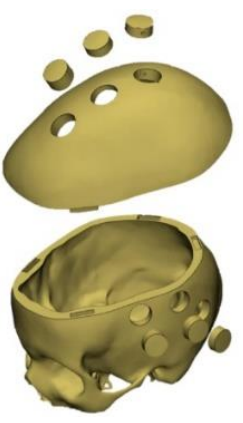

b)



Figura 6. a) Vista de 'ensamble' de modelo para práctica endoscópica, y b) Vista frontal del ensamble.

\subsection{Desarrollo de modelos para enseñanza y educación}

El centro de medicina genética (Newcastle Centre for Life) custodia una de las bases de datos embrionarias más importantes del país, con imágenes médicas embrionarias desde la primera semana de gestación que se utilizan para estudiar el desarrollo de órganos vitales. Dichas imágenes pueden ser visualizadas por medio de software especializado sin embargo estas colecciones no son normalmente digitalizadas y exportadas como modelos físicos (Figura 7).
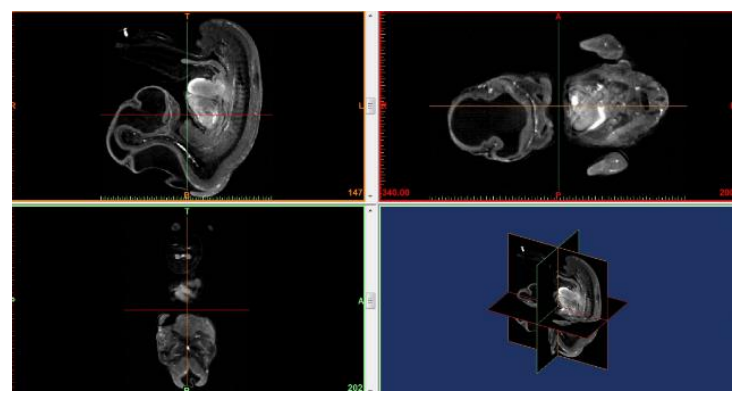

Figura 7. Vistas sagital, coronal y axial de una colección de datos embrionarios. Newcastle Centre for Life [17].

La escuela de ingeniería (UNEW) fue consultada sobre la posibilidad de extraer imágenes y aislar, extraer e imprimir órganos individualmente. Cabe señalar que los técnicos e investigadores del 'Centre for 
Life' no contaban con experiencia previa en diseño, edición e impresión 3D de modelos anatómicos. A partir de una colección de imágenes de un embrión de 5 semanas de gestación fue posible identificar y marcar cada órgano de manera que una vez identificados se pudieran exportar fácilmente a una superficie tridimensional (Figura 8).


Figura 8. Identificación de órganos vitales en $2 D$ (Izquierda) y $3 D$ (Derecha).

Gracias a este ejercicio fue posible por primera vez que la Unidad de Medicina Genética utilizara modelos físicos de representación de órganos embrionarios (ver Figura 9) en eventos de diseminación y clases presenciales, abriendo la puerta a una nueva subactividad en el departamento de radiología.



Figura 9. Modelo generado como una superficie en $3 D$ mediante MIMICS (Izquierda) e impreso en $3 D$ mediante Estereolitografía (Derecha).

\section{CONCLUSIONES}

El despliegue y adopción de tecnologías digitales, relacionadas con el diseño, análisis y fabricación van ligadas a la 'necesidad médica' de distintas Unidades Universitarias y Hospitalarias y en pocos casos son producto de una iniciativa externa. Es decir, a pesar de que un gran catálogo de tecnologías como software de segmentación, diseño CAD de sólidos y superficies, análisis de elementos finitos, impresión 3D y manufactura digital en general, han estado disponibles durante décadas, es solo recientemente que han encontrado eco en la comunidad médica.

Mientras que un departamento o unidad especializada en diseño de implantes y guías médicas, o una unidad interna de desarrollo de dispositivos médicos similares a las ya desplegadas en el Reino Unido en hospitales como Sheffield, Newcastle y Gales, no son realistas en un ambiente hospitalario de México, Colombia o Perú, la tendencia global seguirá marcando su adopción.

De momento las facultades universitarias de ingeniería, particularmente ingenierías Mecánica, Electrónica y Mecatrónica están posicionadas para apoyar desarrollos puntuales a través de colaboraciones con centros sanitarios y a actuar como catalizadores para la adopción de las tecnologías de digitalización.

\section{AGRADECIMIENTOS}

Agradecimiento especial al comité organizador del Primer Simposio Nacional de Prótesis Ortopédicas: México 2020, de dónde se desprende este manuscrito.

\section{REFERENCIAS}

[1] MVI. Informática Nordeste Ltda. La revolución del hospital digital [Online], Available: http://www.mv.com.br/ (accesado Septiembre 2020).

[2] Oxford University Hospitals (OUH), 
Building the Digital Hospital [Online] https://www.ouh.nhs.uk/about/building-thedigital-hospital.aspx, Accessed May 2018. 2018.

[3] Sheffield Teaching Hospitals. Information and Technology Strategy 2020. NHS Foundation trust. 2017.

[4] Wachter, R. "Making IT Work: Harnessing the Power of Health Information Technology to Improve Care in England" 2016.

[5] Kılıç, T. Digital Hospital; An example of best practice. International Journal of Health Services Research and Policy 1(2):52-58

DOI: 10.23884/ijhsrp.2016.1.2.04.

[6] NHS. Morriston Hospital News. [Online] https://sbuhb.nhs.wales/news/newscategories/morriston-hospital-news/, Accessed Sep 2020.

[7] NHS Confederation. Little-known facts about university hospitals. [Online] https://www.nhsconfed.org/, Accessed Sept 2020.

[8] Munguia, J. RAE "Digital Literacy" Indo-UK project. [Online] https://research.ncl.ac.uk/3dprintinglab/resea rch/raedigitalliteracyindo-ukproject/, Access Sep 2020.

[9] Gilliot, J. et.al. Impact of digital literacy on the engineer curriculum. Proceedings of the 6th International CDIO Conference, École Polytechnique, Montréal, June 15-18, 2010.

[10] Chalmers, E.V., G.T. Mcintyre, W. Wang, T. Gillgrass, C.B. Martin, and P.A.
Mossey. "Intraoral 3D Scanning or Dental Impressions for the Assessment of Dental Arch Relationships in Cleft Care: Which Is Superior?" The Cleft Palate-Craniofacial Journal 53, no. 5 (September 2016): 568-77.

[11] Chua et.al.Rapid prototyping: principles and applications (2nd edition) 2003.

[12] Schubert et.al. Innovations in $3 D$ printing: a $3 D$ overview from optics to organs. Br J Ophthalmol 98(2):159-161. 2014.

[13] Munguia J. et.al.Towards the Digital Hospital: From Implant Design to In-Clinic Bio Fabrication. 7th International Conference on the Development of Biomedical Engineering in Vietnam (BME7). BME 2018. IFMBE Proceedings, vol 69. Springer, Singapore.

[14] GOSH. Great Ormond Street Hospital. ICH researchers develop a technique to 'print' healthy new tissue. (Online) Available: https://www.gosh.nhs.uk/news Access Sep 2020.

[15] Jones et.al. Surface modified unit cell lattice structures for optimized secure freeform fabrication. US Patent office. Patent number 9135374.

[16] Ma, et.al. Development of a smart-fit system for CPAP interface selection. Proc IMechE Part H: J Engineering in Medicine 1-10. 2020.

[17] HUDSEN. Human brain gene expression atlas project. Online. https://hudsen.org/ Access Sep 2020. 